\title{
ADDITIVE FUNCTIONALS ON A SPACE OF CONTINUOUS FUNCTIONS. I
}

\author{
BY \\ R. H. CAMERON AND ROSS E. GRAVES
}

1. Introduction. In this paper the authors undertake to obtain a characterization and representation (analogous to the Riesz representation) for a certain class of essentially additive functionals over a space of continuous functions on a finite interval. By a change of scale this interval can be taken to be $I: 0 \leqq t \leqq 1$; and for convenience the functions $x(t)$ are required to vanish at the origin. Thus our space is the Wiener space $C[8]\left({ }^{1}\right)$, and a suitable measure is at once available. We use the term "essentially additive" in the sense of "equivalent to additive," that is, equal almost everywhere to an additive functional. No continuity or even homogeneity is assumed. However, we shall in the hypotheses of our theorems always include a condition of Wiener measurability, and usually a requirement that the functional belong to $L_{2}(C)$. It will be understood that all functionals are finite-valued throughout their domains of definition.

In addition to characterization and representation theorems for essentially additive functionals of class $L_{2}(C)$, we obtain as by-products some even more striking results along different lines. Thus, it is shown that measurable periodic functionals with certain sets of periods must be essentially constant. It is also shown that two measurable additive (or essentially additive) functionals are equal almost everywhere or almost nowhere. This result is an immediate consequence of the following zero-one law.

THeOREM 3. If $M$ is a measurable subset of $C$ which is a linear manifold over the field of rational numbers, then the measure of $M$ is either zero or one.

Another rather interesting theorem which arises as a corollary to our representation theory is the following.

Theorem 7. Every additive (or essentially additive) functional of class $L_{2}(C)$ is essentially homogeneous, and, in fact, essentially linear.

To make this theorem precise, we state the following definition.

Definition 1. A functional on $C$ is "essentially" additive, homogeneous, linear, or constant if it is equal almost everywhere to a functional that is additive, homogeneous, linear, or constant, respectively. Here "linear" means additive and homogeneous, but does not imply boundedness or continuity.

Presented to the International Congress of Mathematicians, September 2, 1950; received by the editors June 23, 1950 .

(1) Numbers in brackets refer to the bibliography at the end of the paper. 
The most important tools which we shall employ in the statements and proofs of our results are the generalized Riemann-Stieltjes integrals of Paley, Wiener, and Zygmund [7]; and the Fourier-Hermite expansion [4] and the translation theorem [2] of Cameron and Martin.

Definition 2. Let $\left\{\phi_{k}\right\}$ be a C.O.N. (complete orthonormal set in the $L_{2}$ sense) on $[0,1]$, each of whose elements is of bounded variation, and let $f(t) \in L_{2}$ on $[0,1]$. Let $f_{n}(t)$ be the $n$th partial sum of the orthogonal development of $f(t)$ in terms of $\left\{\phi_{k}\right\}$; that is, let $f_{n}(t)=\sum_{k=1}^{n} c_{k} \phi_{k}(t)$, where $c_{k}$ $=\int_{0}^{1} f(t) \overline{\phi_{k}(t)} d t$. Then we define the P.W.Z. integral $\int_{0}^{1} f(t) \tilde{d} x(t)$ by the equation

$$
\int_{0}^{1} f(t) \tilde{d} x(t)=\lim _{n \rightarrow \infty} \int_{0}^{1} f_{n}(t) d x(t), \quad x \in C,
$$

whenever the limit on the right exists.

It has been shown by Paley, Wiener, and Zygmund [7] that the limit defining the P.W.Z. integral exists for almost all $x \in C$ and that this limit is essentially independent of the particular choice of the C.O.N. $\left\{\phi_{k}\right\}$ in the sense that if $\left\{\phi_{n}\right\}$ and $\left\{\phi_{n}^{*}\right\}$ are any two C.O.N.'s all of whose elements are of B.V., then $(\phi) \int_{0}^{1} f(t) \tilde{d} x(t)=\left(\phi^{*}\right) \int_{0}^{1} f(t) \tilde{d} x(t)$ for almost all $x \in C$. It has also been shown in [7] that $\int_{0}^{1} f_{n}(t) d x(t)$ converges in the $L_{2}(C)$ mean to $\int_{0}^{1} f(t) \tilde{d} x(t)$, that the P.W.Z. integral is essentially consistent with the ordinary RiemannStieltjes integral when $f(t)$ is of B.V., and that if $G\left(u_{1}, \cdots, u_{n}\right)$ is Lebesgue measurable on $(-\infty, \infty)^{n}$ and $\alpha_{1}(t), \cdots, \alpha_{n}(t)$ are real and O.N. on $[0,1]$, then

$$
\begin{aligned}
\int_{C}^{W} G\left[\int_{0}^{1} \alpha_{1}(t) \tilde{d} x(t), \cdots, \int_{0}^{1} \alpha_{n}(t) \tilde{d} x(t)\right] d W & \\
& =\pi^{-n / 2} \int_{-\infty}^{\infty} \cdots \int_{-\infty}^{\infty} G\left(u_{1}, \cdots, u_{n}\right) e^{-u_{1}^{2}-\cdots-u_{n}^{2}} d u_{1} \cdots d u_{n}
\end{aligned}
$$

where the existence of either side of the above equation as an absolutely convergent integral implies that of the other.

Definition 3. Let

$$
\begin{aligned}
\Psi_{m_{1}}, \cdots, m_{N} & (x)=\coprod_{k=1}^{N} H_{m_{k}}\left[\int_{0}^{1} \alpha_{k}(t) \tilde{d} x(t)\right] \\
& \left(N=1,2, \cdots ; m_{k}=0,1,2, \cdots\right),
\end{aligned}
$$

where the $H_{m}$ 's are the partially normalized Hermite polynomials defined by

$$
H_{m}(u)=(-1)^{m} 2^{-m / 2}(m !)^{-1 / 2} e^{u^{2}} \frac{d^{m}}{d u^{m}}\left(e^{-u^{2}}\right) \quad(m=0,1,2, \cdots),
$$

and where $\left\{\alpha_{k}(t)\right\}$ is a C.O.N. of real-valued functions on $[0,1]$. Then the 
set $\left\{\Psi_{m_{1}, \cdots, m_{N}}(x)\right\}$ will be called the set of Fourier-Hermite polynomials (or functionals).

Wenote that $H_{0}(u)=1, H_{1}(u)=2^{1 / 2} u$, and $\Psi_{m_{1}, \cdots, m N}(x)=\Psi_{m_{1}, \cdots, m_{N}, 0, \cdots, 0}(x)$.

It has been shown by Cameron and Martin [4] that the Fourier-Hermite functionals form a closed (in the $L_{2}(C)$ sense) orthonormal set of functionals. More explicitly, if for $F(x) \in L_{2}(C)$ we define

$$
A_{m_{1}, \cdots, m N}=\int_{C}^{W} F(x) \Psi_{m_{1}, \cdots, m_{N}}(x) d_{W} x,
$$

then

$$
\lim _{N \rightarrow \infty} \int_{C}^{W}\left|F(x)-\sum_{m_{1}, \cdots, m_{N}=0}^{N} A_{m_{1}, \cdots, m_{N}} \Psi_{m_{1}, \cdots, m_{N}}(x)\right|^{2} d_{W} x=0,
$$

and the Parseval equation holds in the sense that

$$
\lim _{N \rightarrow \infty} \sum_{m_{1}, \cdots, m N=0}^{N}\left|A_{m_{1}, \cdots, m_{N}}\right|^{2}=\int_{C}^{W}|F(x)|^{2} d_{W} x .
$$

We shall also need a generalization of the translation theorem for Wiener integrals [2]. In its original form, this theorem states that if $F(x)$ is Wiener measurable on $C$ and $x_{0}(t) \in C$ has at all points of $[0,1]$ a derivative $x_{0}^{\prime}(t)$ which is of B.V. on $[0,1]$, then

$$
\begin{aligned}
& \int_{C}^{W} F\left(x+x_{0}\right) d_{W} x \\
&=\exp \left\{-\int_{0}^{1}\left[x_{0}^{\prime}(t)\right]^{2} d t\right\} \int_{C}^{W} F(x) \exp \left\{2 \int_{0}^{1} x_{0}^{\prime}(t) d x(t)\right\} d_{W} x,
\end{aligned}
$$

in the sense that the existence of either side implies that of the other and the equality. This generalization, due to Maruyama [9] (which we will prove in $\S 2)$, is the following theorem.

THEOREM 1. If $F(x)$ is a Wiener measurable functional on $C$ and $f(t) a$ real-valued function of class $L_{2}$ on $[0,1]$, then

$$
\begin{aligned}
\int_{C}^{W} F(x(\cdot) & \left.+\int_{0}^{(\cdot)} f(u) d u\right) d_{W} x \\
& =\exp \left\{-\int_{0}^{1}[f(t)]^{2} d t\right\} \int_{C}^{W} F(x) \exp \left\{2 \int_{0}^{1} f(t) \tilde{d} x(t)\right\} d_{W} x
\end{aligned}
$$

in the sense that the existence of either side implies that of the other and the equality.

Some of the theorems on additive functionals which we shall prove in- 
volve spaces other than the space $C$ which we introduce at this point.

Definition 4 . Let $L_{p}^{(n)}([a, b])$ be the set of absolutely continuous functions on $[a, b]$ which have $n-1$ absolutely continuous derivatives and an $n$th derivative of class $L_{p}$ on $[a, b]$.

2. The translation theorem. For the proof of the generalized translation theorem we require the following lemma.

Lemma 1. Let $f_{n}(t)$ be real-valued and of $B . V$. on $[0,1](n=1,2, \cdots)$, and let $f_{n}(t)$ converge in the $L_{2}$ mean on $[0,1]$ as $n \rightarrow \infty$. Then for any real number $\lambda, \exp \left\{\lambda \int_{0}^{1} f_{n}(t) d x(t)\right\}$ converges in the $L_{2}$ mean on $C$ as $n \rightarrow \infty$.

Proof. By a simple calculation, using (1.1), we obtain

$$
\begin{aligned}
& \int_{C}^{W}\left[\exp \left\{\lambda \int_{0}^{1} f_{m}(t) d x(t)\right\}-\exp \left\{\lambda \int_{0}^{1} f_{n}(t) d x(t)\right\}\right]^{2} d_{W} x \\
&=\int_{C}^{W} \exp \left\{2 \lambda \int_{0}^{1} f_{m}(t) d x(t)\right\} d_{W} x \\
&-2 \int_{C}^{W} \exp \left\{\lambda \int_{0}^{1}\left[f_{m}(t)+f_{n}(t)\right] d x(t)\right\} d_{W} x \\
&+\int_{C}^{W} \exp \left\{2 \lambda \int_{0}^{1} f_{n}(t) d x(t)\right\} d_{W} x \\
&= \exp \left\{\lambda^{2} \int_{0}^{1}\left[f_{m}(t)\right]^{2} d t\right\}-2 \exp \left\{\frac{\lambda^{2}}{4} \int_{0}^{1}\left[f_{m}(t)+f_{n}(t)\right]^{2} d t\right\} \\
&+\exp \left\{\lambda^{2} \int_{0}^{1}\left[f_{n}(t)\right]^{2} d t\right\} .
\end{aligned}
$$

Since each exponent in the last member approaches the same limit as $m$ and $n$ approach $\infty$, the lemma follows.

Proof of Theorem 1. Case I. Let $F(x)$ be bounded and continuous in the uniform topology. Let $\left\{\alpha_{k}(t)\right\}$ be a C.O.N. for which each element $\alpha_{k}(t)$ is real-valued, continuous, and B.V. Let $f_{n}(t)=\sum_{k=1}^{n} \alpha_{k}(t) \int_{0}^{1} f(u) \alpha_{k}(u) d u$, and apply the original translation theorem with $x_{0}(t)=\int_{0}^{t} f_{n}(u) d u$. Then we have

$$
\begin{aligned}
& \int_{C}^{W} F\left(x(\cdot)+\int_{0}^{(\cdot)} f_{n}(u) d u\right) d_{W} x \\
& \quad=\exp \left\{-\int_{0}^{1}\left[f_{n}(u)\right]^{2} d u\right\} \int_{C}^{W} F(x) \exp \left\{2 \int_{0}^{1} f_{n}(t) d x(t)\right\} d_{W} x,
\end{aligned}
$$

and the measurability of the integrand of the left member is established.

Since $\int_{0}^{1} f_{n}(t) d x(t) \rightarrow \int_{0}^{1} f(t) \tilde{d} x(t)$ for almost all $x$ as $n \rightarrow \infty$, it follows from Lemma 1 that the right member of (2.1) approaches the right member of 
(1.2) as $n \rightarrow \infty$. Moreover, since $\int_{0}^{t} f_{n}(u) d u$ approaches $\int_{0}^{t} f(u) d u$ uniformly and $F(x)$ is bounded and continuous in the uniform topology, the left member of (2.1) approaches the left member of (1.2), and Case I is established.

Case II. The proof of Theorem 1 is completed by extending Case I to the general case. This extension is word for word identical to the corresponding extension to the general case in the proof of the original translation theorem. (See pp. 391-392 of [2].)

Corollary 1. Let $f(t)$ be real-valued and of class $L_{2}$ on $[0,1]$, let $T$ be the translation

$$
T(x)=x(t)-\int_{0}^{t} f(u) d u,
$$

and let $M$ be any measurable subset of $C$. Then $T(M)$ is measurable and

$$
m_{W}(T(M))=\exp \left\{-\int_{0}^{1}[f(t)]^{2} d t\right\} \int_{M}^{W} \exp \left\{2 \int_{0}^{1} f(t) \tilde{d} x(t)\right\} d_{W} x .
$$

CoROLlary 2. If $f(t)$ is real-valued and of class $L_{2}$ on $[0,1]$, the translation (2.2) takes sets of positive measure into sets of positive measure.

3. Statement of the fundamental lemma. In this section we state a lemma which is the basis for most of the results of this paper. Since the details are rather lengthy, we defer the proof until $\S 8$. In order to make the lemma apply both to periodic functionals and to additive functionals, we have introduced the constant $K$ which is zero in the periodic case and unity in the additive case.

Lemma 2. Let $\left\{\alpha_{k}(t)\right\}$ be a C.O.N. of real-valued functions on $[0,1], \tau \neq 0$ a fixed real number, $\Lambda$ a set of real numbers which has at least one limit point $\lambda_{0}$ such that $\left|\lambda_{0}\right|<\sup _{\lambda \in \Lambda}|\lambda|$, and

$$
\beta_{k}(t)=\tau \int_{0}^{t} \alpha_{k}(u) d u
$$

Let $F(x)$ be defined on $C$, of class $L_{2}(C)$, and such that for $\lambda \in \Lambda$ and $x \in C$,

$$
F\left(x+\lambda \beta_{k}\right)=F(x)+\lambda K F\left(\beta_{k}\right),
$$

where $K$ is a constant. Then the Fourier-Hermite series of $F(x)$ with respect to $\left\{\alpha_{k}(t)\right\}$ contains only a constant and first degree terms. Specifically $\left(^{2}\right)$

$$
F(x) \sim \text { const. }+K \tau^{-1} \sum_{k=1}^{\infty} F\left(\beta_{k}\right) \int_{0}^{1} \alpha_{k}(t) \tilde{d} x(t) .
$$

(2) Actually it is seen without difficulty that this series converges for almost all $x$, as well as in the $L_{2}(C)$ sense (see [6]). We do not bother to establish this refinement as it is unnecessary for our purposes. 
Moreover

$$
K F\left(\beta_{k}\right)=2 \tau \int_{C}^{W} F(x)\left\{\int_{0}^{1} \alpha_{k}(t) \tilde{d} x(t)\right\} d_{W} x .
$$

Corollary. If $F(x)$ is additive and of class $L_{2}(C)$, then

$$
F(x)=2 \int_{C}^{W} F(y)\left\{\int_{0}^{1} x^{\prime}(t) \tilde{d} y(t)\right\} d_{W} y ; \quad x \in L_{2}^{\prime}(I) \cdot C .
$$

Proof. Since $F(x)$ is additive, (3.2) holds with $\Lambda$ the set of rational numbers and $K=1$. If $x(t) \not \equiv 0$, so that $\int_{0}^{1}\left[x^{\prime}(t)\right]^{2} d t \neq 0$, we can choose

$$
\tau=\left(\int_{0}^{1}\left[x^{\prime}(u)\right]^{2} d u\right)^{1 / 2}, \quad \alpha_{1}(t)=x^{\prime}(t)\left(\int_{0}^{1}\left[x^{\prime}(u)\right]^{2} d u\right)^{-1 / 2}
$$

in the lemma. The result now follows from (3.4). The case $x(t) \equiv 0$ is trivial.

4. Periodic functionals and $r$-modules. As a consequence of the fundamental lemma, we now obtain a theorem which shows that if a functional has too many periods, it is essentially constant.

THEOREM 2. Let $\left\{\alpha_{k}(t)\right\}$ be a C.O.N. of real-valued functions, $\Lambda$ a set of real numbers having a finite limit point, and $\beta_{k}(t)=\int_{0}^{t} \alpha_{k}(u) d u$. Let $F(x)$ be measurable on $C$ and such that

$$
F\left(x+\lambda \beta_{k}\right)=F(x) \quad(\lambda \in \Lambda ; k=1,2, \cdots) .
$$

Then $F(x)=$ const. almost everywhere on $C$.

Proof. Case I. $F(x) \in L_{2}(C)$. We apply the fundamental lemma with $K=0$ and $\tau=1$, noting that if (4.1) is satisfied for $\lambda$, it is also satisfied for $n \lambda$. The result follows from (3.3).

Case II. $F(x)$ measurable. Define

$$
F_{n}(x)=\left\{\begin{array}{cll}
F(x) & \text { if } & |F(x)| \leqq n \\
0 & \text { if } & |F(x)|>n
\end{array}\right.
$$

Then $F_{n}(x) \in L_{2}(C)$ and $F_{n}\left(x+\lambda \beta_{k}\right)=F_{n}(x)$. From Case I, $F_{n}(x)=$ const. almost everywhere, and hence the same is true of $F(x)=\lim _{n \rightarrow \infty} F_{n}(x)$.

We now show by counterexample that even if $\Lambda$ is the set of all real numbers the hypotheses of Theorem 2 are not sufficient to imply that $F(x)$ is constant everywhere on $C$.

COUNTEREXAMPLE. There exists a bounded measurable functional which has as periods all functions which satisfy a Lipschitz condition of order 1/4, but which is not identically constant on $C$.

For let $M$ be the set of all functions of $C$ which satisfy a Lipshitz condition of order $1 / 4$. It is known [8] that $m_{W}(M)=1$. Define $F(x)=\chi_{M}(x)$, where $\chi_{M}(x)$ is the characteristic functional of $M$. It is clear that this functional has 
the prescribed properties.

By use of Theorem 2, we establish a new form of the zero-one law.

Definition 5. We call a subset $M$ of $C$ an $r$-module if for all rational numbers $r_{1}, r_{2}$

$$
x, y \in M \rightarrow r_{1} x+r_{2} y \in M .
$$

ThEOREM 3. Every measurable r-module has Wiener measure zero or one. lemma.

The proof of this theorem depends upon the following rather curious

Lemma 3. If $M$ is a measurable $r$-module and $m_{W}(M)>0$, then $L_{2}^{\prime}(I) \cdot C \subset M$.

Proof. Suppose there is a function $y_{0} \in L_{2}^{\prime}(I) \cdot C$ such that $y_{0} \notin M$. Define the translated set $M_{\lambda}$ by

$$
M_{\lambda}=\varepsilon_{x}\left\{x=y+\lambda y_{0} ; y \in M\right\}
$$

Then for any two distinct rational numbers $r_{1}, r_{2}$, we have $M_{r_{1}} \cdot M_{r_{2}}=0$. Upon applying Corollary 1 of the generalized translation theorem to $M_{\lambda}$, we see that $M_{\lambda}$ is measurable and of positive measure. Moreover

$$
\text { (4.2) } m_{W}\left(M_{\lambda}\right)=\exp \left\{-\lambda^{2} \int_{0}^{1}\left[y_{0}^{\prime}(t)\right]^{2} d t\right\} \int_{M}^{W} \exp \left\{-2 \lambda \int_{0}^{1} y_{0}^{\prime}(t) \tilde{d} y(t)\right\} d_{W} y .
$$

As the functional $\exp \left\{\mu\left|\int_{0}^{1} y_{0}^{\prime}(t) \tilde{d} y(t)\right|\right\}$ is summable on $C$ for any $\mu$, we conclude from the theorem of dominated convergence and (4.2) that $m_{W}\left(M_{\lambda}\right)$ is a continuous function of $\lambda$. We now consider the sequence $\left\{m_{W}\left(M_{1 / n}\right)\right\}$. By continuity and the fact that $M_{0}=M$, we see that $m_{W}\left(M_{1 / n}\right) \rightarrow m_{W}(M)$; hence for some $\epsilon>0, m_{W}\left(M_{1 / n}\right) \geqq \epsilon$ for all $n$. As $M_{1 / n} \cdot M_{1 / m}=0(m \neq n)$, we must have

$$
\sum_{n=1}^{\infty} m_{W}\left(M_{1 / n}\right)=m_{W}\left(\sum_{n=1}^{\infty} M_{1 / n}\right) \leqq m_{W}(C)=1,
$$

and this is impossible in view of the fact that $m_{W}\left(M_{1 / n}\right) \geqq \epsilon$.

Proof of Theorem 3. Let $\left\{\alpha_{k}(t)\right\}$ be a real C.O.N. Then if $m_{W}(M)>0$, $\beta_{k}(t) \in M$, where $\beta_{k}(t)=\int_{0}^{t} \alpha_{k}(u) d u$. Further, if $\lambda$ is any rational number, then

$$
\chi_{M}\left(x+\lambda \beta_{k}\right)=\chi_{M}(x) \quad(x \in C ; k=1,2, \cdots),
$$

where $\chi_{M}(x)$ is the characteristic functional of $M$. By Theorem 2, $\chi_{M}(x)$ $=$ const. almost everywhere. As $m_{W}(M)>0$, we must have $\chi_{M}(x)=1$ almost everywhere on $C$, whence $m_{W}(M)=1$.

5. Uniqueness properties of additive functionals. Our zero-one theorem of the last section yields immediately the following remarkable uniqueness theorem. 
THEOREM 4. Two measurable additive functionals are equal either almost everywhere or almost nowhere.

For the set where they agree is a measurable $r$-module.

Another type of uniqueness theorem is obtained as an immediate consequence of the corollary to the fundamental lemma.

TheOREM 5. If $F(x)$ is of class $L_{2}(C)$ and equivalent to an additive functional $F^{*}(x)$, then $F^{*}(x)$ is uniquely determined on $L_{2}^{\prime}(I) \cdot C$ by the equation

$$
F^{*}(x)=2 \int_{C}^{W} F(y)\left\{\int_{0}^{1} x^{\prime}(t) d y(t)\right\} d_{W} y .
$$

In consequence, $F^{*}(x)$ is linear on $L_{2}^{\prime}(I) \cdot C$.

THEOREM 5a. If $F(x)$ and $G(x)$ are additive on $C$ and equal almost everywhere on $C$, then $F(x)=G(x)$ throughout $L_{2}^{\prime}(I) \cdot C$.

The result follows by applying Theorem 5 to $F(x)-G(x)$. Note that we do not require that $F(x)$ and $G(x)$ be measurable.

6. Characterization and representation theorems. We now characterize the essentially additive functionals of class $L_{2}(C)$.

Theorem 6. A necessary and sufficient condition that a functional of class $L_{2}(C)$ be essentially additive is that its Fourier-Hermite development with respect to some real C.O.N. should contain only first degree terms. Moreover if this condition is satisfied for some real C.O.N. then it is satisfied for every real C.O.N.

Proof. The necessity of the condition follows readily from the fundamental lemma if we take $K=\tau=1, \Lambda$ the set of rational numbers, and apply the lemma to an equivalent additive functional $F^{*}(x)$. The constant term vanishes because the average of an odd summable functional is zero. It is also clear from the lemma that the conclusion holds not just for one real C.O.N., but for every real C.O.N.; that is, if $F(x) \in L_{2}(C)$ and $F(x)$ is essentially additive, then for every real C.O.N. $\left\{\alpha_{k}(t)\right\}$ the F.H. development of $F(x)$ contains only first degree terms.

The sufficiency of the condition follows quite readily from a theorem of Kolmogoroff [6]. However, a proof more in the spirit of the present paper is the following. Let $F(x)$ be a functional of class $L_{2}(C)$ whose F.H. development with respect to the real C.O.N. set $\left\{\alpha_{k}(t)\right\}$ contains only first degree terms. Let us select a C.O.N. set of functions of B.V., $\left\{\gamma_{j}(t)\right\}$, and let the P.W.Z. integrals $\int_{0}^{1} \alpha_{k}(t) \tilde{d} x(t)$ be defined with respect to $\left\{\boldsymbol{\gamma}_{j}\right\}$. Then each $\int_{0}^{1} \alpha_{k}(t) \tilde{d} x(t)$ exists and is linear for $x$ on a linear subspace $E_{k}$ of $C$ of measure 1 , and they all exist and are linear on the linear subspace $E=\prod_{k=1}^{\infty} E_{k}$ which is also of measure 1. Now if $A_{m_{1}}, \cdots, m_{N}$ are the coefficients of the F.H. development of $F(x)$ with respect to $\left\{\alpha_{k}\right\}$, by hypothesis all the $A$ 's are zero except those of 
the form $A_{0,0, \cdots, 0,1}=a_{k}$, where the subscript 1 on the $A$ occurs in the $k$ th place. Thus

$$
F(x)=\underset{k \rightarrow \infty}{\operatorname{L.I.M}} \sum_{j=1}^{k} 2^{1 / 2} a_{j} \int_{0}^{1} \alpha_{j}(t) \tilde{d} x(t),
$$

and by the corollary to the Riesz-Fischer theorem, we can extract a subsequence of the partial sums, say for $k=N_{n}$, such that $\lim _{n \rightarrow \infty}$ $\sum_{j=1}^{N_{n}} 2{ }^{1 / 2} a_{j} \int_{0}^{1} \alpha_{j}(t) \bar{d} x(t)$ exists almost everywhere on $C$. Let $E^{*}$ be the subset of $E$ where this limit exists. Then $E^{*}$ is a linear subspace of $C$ of measure unity, and the functional

$$
F^{*}(x)=\lim _{n \rightarrow \infty} \sum_{j=1}^{N_{n}} 2^{1 / 2} a_{j} \int_{0}^{1} \alpha_{j}(t) \tilde{d} x(t)
$$

exists and is linear everywhere on $E^{*}$. We now extend $F^{*}(x)$ from $E^{*}$ to a linear functional $F^{* *}(x)$ defined everywhere on $C$. But as $F^{* *}(x)=F(x)$ almost everywhere on $C$, it follows that $F(x)$ is essentially linear and hence essentially additive. The final assertion of the theorem follows at once from this suffciency result and the immediately preceding strong form of the necessity result.

Since our proof of sufficiency established not only the essential additivity of $F(x)$, but actually its essential linearity, we have also established the following theorem.

THEOREM 7. If a functional of class $L_{2}(C)$ is essentially additive, it is essentially linear.

In a sense, Theorem 7 is a best possible result for $L_{2}(C)$, as we show by the following counterexample.

COUNTEREXAMPLE. There exists a functional belonging to $L_{p}(C)$ for all $p>0$ which is defined and additive everywhere on $C$, but which is not homogeneous on $C$ (and hence, of course, not linear on $C$ ).

Let $S$ be the linear subspace of $C$ consisting of all functions which satisfy for some $h(x)$ the Lipshitz condition of order $1 / 4$,

$$
\left|x\left(t_{1}\right)-x\left(t_{2}\right)\right| \leqq h(x)\left|t_{1}-t_{2}\right|^{1 / 4} \quad \text { for all } t_{1}, t_{2} \in[0,1] .
$$

It is well known that $m_{W}(S)=1$. Let $\xi(t)=t^{1 / 8}$, so that $\xi \notin S$. Let $S^{*}$ be defined by

$$
S^{*}=\varepsilon_{y}\left\{y=x+\left(r_{1}+r_{2} \pi\right) \xi ; x \in S ; r_{1}, r_{2} \text { rational }\right\},
$$

and define $F(x)$ on $S^{*}$ by

$$
F\left(x+\left(r_{1}+r_{2} \pi\right) \xi\right)=x(1)+r_{1},
$$

where $x \in S$ and $r_{1}$ and $r_{2}$ are rational. 
Then $F(x)$ is uniquely defined and additive on $S^{*}$, but not homogeneous there. As $S^{*}$ is a linear vector space over the field of rational numbers, $F(x)$ can be extended to a functional $F^{*}(x)$ additive over the whole of $C$ which agrees with $F(x)$ on $S^{*}$. It is clear that $F^{*}(x) \in L_{p}(C)$ for each $p>0$, while it is not homogeneous, as $F^{*}(\xi)=1, F^{*}(\pi \xi)=0$.

We now establish the representation theorem for essentially additive functionals of class $L_{2}(C)$.

THEOREM 8. A necessary and sufficient condition that $F(x)$ be essentially additive and of class $L_{2}(C)$ is that it be of the form

$$
F(x)=\int_{0}^{1} f(t) \tilde{d} x(t)
$$

for almost all $x \in C$, where $f(t) \in L_{2}(I)$. Moreover, if $F(x)$ is essentially additive and of class $L_{2}(C)$, the function $f(t)$ of $(6.1)$ is given by

$$
f(t)=2 \frac{d}{d t} \int_{C}^{W} F(x) x(t) d_{W} x
$$

for almost all $t \in[0,1]$.

The sufficiency of the condition follows from the fact that the series defining the P.W.Z. integral converges in the $L_{2}(C)$ mean, and that any C.O.N. set of functions of B.V. makes $\int_{0}^{1} f(t) \tilde{d} x(t)$ additive (in fact linear) on a linear subspace of $C$ of measure 1. Thus $\int_{0}^{1} f(t) \tilde{d} x(t) \in L_{2}(C)$ and since this functional is defined almost everywhere, it can be extended to a linear functional on $C$.

To establish the necessity of the condition, we note that as $F(x)$ is essentially additive and of class $L_{2}(C)$, by Theorem 6 its F.H. series with respect to any real C.O.N. contains only first degree terms. We choose a particular real C.O.N. set of functions of B.V., say $\left\{\alpha_{k}(t)\right\}$, for the remainder of the proof. Let

$$
F(x) \sim \sum_{k=1}^{\infty} 2^{1 / 2} a_{k} \int_{0}^{1} \alpha_{k}(t) d x(t),
$$

where $a_{k}$ has the same meaning as in the proof of Theorem 6. As $\sum_{k=1}^{\infty}\left|a_{k}\right|^{2}$ converges, there is a function $f(t) \in L_{2}(I)$ such that $f(t) \sim \sum_{k=1}^{\infty} 2^{1 / 2} a_{k} \alpha_{k}(t)$. By definition of the P.W.Z. integral

$$
\int_{0}^{1} f(t) \tilde{d} x(t)=\sum_{k=1}^{\infty} 2^{1 / 2} a_{k} \int_{0}^{1} \alpha_{k}(t) d x(t),
$$

where the series converges almost everywhere on $C$ and converges in the $L_{2}$ mean on $C$, so that $F(x)=\int_{0}^{1} f(t) \tilde{d} x(t)$ for almost all $x \in C$. Now by mean convergence and (1.1) 


$$
\begin{aligned}
\int_{C}^{W} F(x) x(t) d_{W} x & =\sum_{k=1}^{\infty} 2^{1 / 2} a_{k} \int_{C}^{W}\left\{\int_{0}^{1} \alpha_{k}(u) d x(u)\right\} x(t) d_{W} x \\
& =\sum_{k=1}^{\infty} 2^{1 / 2} a_{k}\left(\frac{1}{2} \int_{0}^{t} \alpha_{k}(u) d u\right)=\frac{1}{2} \int_{0}^{t} f(u) d u,
\end{aligned}
$$

from which (6.2) follows at once.

7. Additive functionals on $L_{2}^{\prime \prime}$. In the previous sections we have usually assumed that our additive functionals were of class $L_{2}(C)$. It is of some interest to see whether any results can be obtained if we replace the $L_{2}(C)$ condition by mere measurability. We shall show in this section that additivity and measurability on $C$ imply certain results on the subspace $L_{2}^{\prime \prime}(I) \cdot J$, where $J$ is the set of elements $x \in C$ such that $x^{\prime}(1)=0$. We first prove the following lemma.

Lemma 4. Let $F(x)$ be additive and measurable on $C$, and let $S$ be the subset of $L_{2}^{\prime \prime}(I) \cdot C$ such that $x^{\prime}(1)=0$ and $\int_{0}^{1}\left[x^{\prime \prime}(t)\right]^{2} d t<1$. Then $F(x)$ is bounded on $S$.

Proof. Suppose the lemma false. Then there exists a sequence $\left\{x_{n}\right\}$ of elements of $S$ sueh that $F\left(x_{n}\right)<F\left(x_{n+1}\right)$ and $\lim _{n \rightarrow \infty} F\left(x_{n}\right)=+\infty$. Let $T$ be the subset of $C$ such that $\int_{0}^{1}[x(t)]^{2} d t<1$. Define

$$
\begin{aligned}
U & =\varepsilon_{x}\left\{x=x^{*}+x^{* *} ; x^{*} \in S, x^{* *} \in T\right\}, \\
E_{n} & =\varepsilon_{x}\left\{F(x)<\frac{1}{2} F\left(x_{n}\right), x \in T\right\}, \\
G_{n} & =\varepsilon_{x}\left\{F(x) \geqq \frac{1}{2} F\left(x_{n}\right), x \in U\right\} .
\end{aligned}
$$

It is clear from these definitions that $G_{n} \supset G_{n+1}, T \subset U$ (as $0 \in S$ ), and also that $T \subset E_{n}+G_{n}$. Define also

$$
H_{n}=\varepsilon_{x}\left\{x=x_{n}-y, y \in E_{n}\right\} .
$$

For any set $E \subset C$ we define

$$
-E=\varepsilon_{x}\{x=-y, y \in E\} .
$$

By reference to (7.3) we see that $H_{n}$ is the translation of $-E_{n}$ by the function $x_{n}$.

Now if $y \in E_{n}, F\left(x_{n}-y\right)=F\left(x_{n}\right)-F(y)>F\left(x_{n}\right)-F\left(x_{n}\right) / 2=F\left(x_{n}\right) / 2$. More. over, $x_{n}-y \in U$, since $y \in T$ (hence $-y \in T$ ) and $x_{n} \in S$. From these observations and (7.2) we conclude that $x_{n}-y \in G_{n}$. Thus we have $H_{n} \subset G_{n}$. Hence by Corollary 1 to the generalized translation theorem and the fact that the Wiener measure is invariant under reflection 


$$
\begin{aligned}
m_{W}^{(i)}\left(G_{n}\right) & \geqq m_{W}\left(H_{n}\right) \\
& =\exp \left\{-\int_{0}^{1}\left[x_{n}^{\prime}(t)\right]^{2} d t\right\} \int_{-E_{n}}^{W} \exp \left\{-2 \int_{0}^{1} x_{n}^{\prime}(t) d x(t)\right\} d_{W} x \\
& =\exp \left\{-\int_{0}^{1}\left[x_{n}^{\prime}(t)\right]^{2} d t\right\} \int_{E_{n}}^{W} \exp \left\{2 \int_{0}^{1} x_{n}^{\prime}(t) d x(t)\right\} d_{W} x .
\end{aligned}
$$

Since $x_{n} \in S, \int_{0}^{1}\left[x_{n}^{\prime \prime}(t)\right]^{2} d t<1$. Also

$$
\begin{aligned}
\left|x_{n}^{\prime}(t)\right| & =\left|\int_{t}^{1} x_{n}^{\prime \prime}(u) d u\right| \\
& \leqq \int_{0}^{1}\left|x_{n}^{\prime \prime}(t)\right| d t<1 .
\end{aligned}
$$

As $E_{n} \subset T$ we have for $x \in E_{n}, \int_{0}^{1}[x(t)]^{2} d t<1$. Hence for $x \in E_{n}$,

$$
\begin{aligned}
\left|\int_{0}^{1} x_{n}^{\prime}(t) d x(t)\right| & =\left|\int_{0}^{1} x_{n}^{\prime \prime}(t) x(t) d t\right| \\
& \leqq\left(\int_{0}^{1}\left[x_{n}^{\prime \prime}(t)\right]^{2} d t\right)^{1 / 2}\left(\int_{0}^{1}[x(t)]^{2} d t\right)^{1 / 2}<1 .
\end{aligned}
$$

Upon applying these estimates to (7.4) we find

$$
m_{W}^{(i)}\left(G_{n}\right) \geqq e^{-3} m_{W}\left(E_{n}\right) .
$$

It is also known $[1,3]$ that the Wiener measure of any Hilbert neighborhood of the origin is positive, so that $m_{W}(T)>0$. Thus, as $T \subset E_{n}+G_{n}, m_{W}\left(E_{n}\right)$ $+m_{W}^{(i)}\left(G_{n}\right) \geqq m_{W}(T)$, and upon taking account of (7.5) we obtain

$$
\begin{aligned}
m_{W}^{(i)}\left(G_{n}\right) & \geqq\left(1+e^{3}\right)^{-1} m_{W}(T) \\
& >0 .
\end{aligned}
$$

We have seen that $G_{n} \supset G_{n+1}$. Let $\lim G_{n}=G_{\infty}$. Then, since for decreasing sequences of sets of finite measure the inner measure of the limit is the limit of the inner measure, upon taking account of (7.6) we find that $m_{W}^{(i)}\left(G_{\infty}\right)>0$, so that $G_{\infty} \neq 0$. Thus on $G_{\infty}, F(x)=+\infty$, contrary to hypothesis.

THEOREM 9. Let $F(x)$ be additive and measurable on $C$. Then there exists a constant $B$ such that

$$
|F(x)| \leqq B\left(\int_{0}^{1}\left[x^{\prime \prime}(t)\right]^{2} d t\right)^{1 / 2} \quad \text { for } x \in L_{2}^{\prime \prime}(I) \cdot J
$$

Moreover, $F(x)$ is homogeneous on $L_{2}^{\prime \prime}(I) \cdot J$. 
Proof. Let $B$ be the (finite) bound for $|F(x)|$ on $S$, where $S$ is as in the preceding lemma. Let $\eta$ be a rational number greater than 1 and let $x \in L_{2}^{\prime \prime}(I)$ . $J$. Without loss of generality we may assume that $x(t) \neq 0$, and thus that $\int_{0}^{1}\left[x^{\prime \prime}(t)\right]^{2} d t \neq 0$. Let $r>0$ be a rational number such that

$$
r^{2} \leqq \int_{0}^{1}\left[x^{\prime \prime}(t)\right]^{2} d t<r^{2} \eta^{2} .
$$

Then $(r \eta)^{-1} x(t) \in S$, so that $\left|F\left[(r \eta)^{-1} x\right]\right| \leqq B$. Then by (7.8) and the rational homogeneity of $F(x)$

$$
\begin{aligned}
|F(x)| & \leqq r \eta B \\
& \leqq \eta B\left(\int_{0}^{1}\left[x^{\prime \prime}(t)\right]^{2} d t\right)^{1 / 2} .
\end{aligned}
$$

As (7.9) holds for all rational $\eta>1,(7.7)$ follows. Homogeneity of $F(x)$ now follows immediately, as (7.7) asserts that $F(x)$ is continuous on $L_{2}^{\prime \prime}(I) \cdot J$ with respect to the topology defined by $\|x\|=\left(\int_{0}^{1}\left[x^{\prime \prime}(t)\right]^{2} d t\right)^{1 / 2}$.

The above theorem can be rephrased as

THEOREM 9a. If $F(x)$ is additive and measurable on $C$, then the functional

$$
G(f)=F\left[\int_{0}^{(\cdot)} d v \int_{1}^{v} f(u) d u\right]
$$

is a continuous linear functional on the Hilbert space of real-valued functions of class $L_{2}$ on the unit interval.

The preceding theorem yields an interesting method for the construction of nonmeasurable functionals.

TheOREM 10. Let $\Theta$ be the linear subspace of $L_{2}^{\prime \prime}(I) \cdot J$ such that $x^{\prime \prime}(t)$ is continuous for all $t \in I$, and let $t_{0} \in I$. On $\Theta$ define $F(x)=x^{\prime \prime}\left(t_{0}\right)$. Then every additive extension of $F(x)$ to the whole of $C$ is nonmeasurable.

Proof. Suppose that $F^{*}(x)$ were an additive and measurable extension of $F(x)$ to the whole of $C$. By Theorem 9 there exists a number $B$ such that

$$
\left|F^{*}(x)\right| \leqq B\left(\int_{0}^{1}\left[x^{\prime \prime}(t)\right]^{2} d t\right)^{1 / 2}, \quad x \in \Theta .
$$

Define $x_{n}(t)=\int_{0}^{t} d v \int_{1}^{0} e^{-n\left|u-t_{0}\right|} d u$. Then $x_{n} \in \Theta$ and $x_{n}{ }^{\prime}(t)=e^{-n\left|t-t_{0}\right|}$, so that we must have $F^{*}\left(x_{n}\right)=1$. Thus $1 \leqq B\left(\int_{0}^{1} e^{-2 n\left|t-t_{0}\right|} d t\right)^{1 / 2}$, which is impossible as $\int_{0}^{1} e^{-2 n\left|t-t_{0}\right|} d t \rightarrow 0$ as $n \rightarrow \infty$.

8. Proof of the fundamental lemma. Formulas (3.3) and (3.4) will be established (under the hypotheses of the lemma) by expanding both members of (3.2) in F.H. series and comparing coefficients. This comparison will 
show that all but the constant and the linear terms drop out of the expansion.

The expansion of the right member of (3.2) is trivial, as all the coefficients are the same as those of $F(x)$ except for the constant term, which is increased by $\lambda K F\left(\beta_{k}\right)$. To expand the left member of (3.2) (which is of class $L_{2}(C)$ since it is equal to the right member), let

$$
F\left(x+\lambda \beta_{k}\right) \sim \sum B_{m_{1}}, \cdots, m_{n} \Psi_{m_{1}}, \cdots, m_{n}(x) .
$$

If it becomes necessary to indicate the dependence of the $B$ 's on $k$ and $\lambda$, we shall write

$$
B_{m_{1}, \cdots, m_{n}}={ }_{(k)} B_{m_{1}, \cdots, m_{n}}(\lambda) .
$$

Then

$$
\begin{aligned}
B_{m_{1}, \cdots, m_{n}}= & \int_{C}^{W} F\left(x+\lambda \beta_{k}\right) \Psi_{m_{1}, \cdots, m_{n}}(x) d_{W} x \\
= & \exp \left\{-\tau^{2} \lambda^{2} \int_{0}^{1}\left[\alpha_{k}(t)\right]^{2} d t\right\} \int_{C}^{W} F(x) \Psi_{m_{1}}, \cdots, m_{n}\left(x-\lambda \beta_{k}\right) \\
& \cdot \exp \left\{2 \tau \lambda \int_{0}^{1} \alpha_{k}(t) \tilde{d} x(t)\right\} d_{W} x
\end{aligned}
$$

by Theorem 1 .

Since the addition of extra zeros as subscripts after the subscripts of $B_{m_{1}, \cdots, m_{n}}$ does not change the value of this coefficient, we may assume without loss of generality that $n \geqq k$, and we make this assumption henceforth. As a matter of fact, we get all the information we need from the special case $m_{k}=0$; and we therefore also make this assumption. We now compute the integral in the last member of (8.2) by expanding the coefficient of $F(x)$ in a F.H. series thus:

$$
\begin{aligned}
\exp \left\{2 \tau \lambda \int_{n}^{1} \alpha_{k}(t) \tilde{d} x(t)\right\} \Psi_{m_{1}, \cdots, m_{n}}(x- & \left.\lambda \beta_{k}\right) \\
& \sim \sum D_{\mu_{1}, \cdots, \mu_{\nu}} \Psi_{\mu_{1}, \cdots, \mu_{\nu}}(x),
\end{aligned}
$$

where

$$
D_{\mu_{1}, \cdots, \mu_{\nu}}={ }_{(k)} D_{\mu_{1}, \cdots, \mu_{\nu}}^{m_{1}, \cdots, m_{n}}(\lambda) .
$$

The expansion exists, since the left member of (8.3) is of class $L_{2}(C)$ because each factor is of class $L_{4}(C)$. Again, we can increase $\nu$ by adding zero subscripts if necessary, to obtain without loss of generality $\nu \geqq n \geqq k$. We proceed to calculate the coefficients $D_{\mu_{1}, \ldots, \mu_{\nu}}$ by making a translation (Theorem 1 ) and using the Wiener formula (1.1) to evaluate the Wiener integral depending on $\nu$ linear functionals. We also use the orthonormality of the $\left\{\alpha_{j}\right\}$ and 
the $\{\Psi\}$, and the fact that $m_{k}=0$. Thus

$$
\begin{aligned}
& { }_{(k)} D_{\mu_{1}, \cdots, \mu_{v}-1,0, m_{k}+1, \cdots, m_{n}}^{\left.m_{1}, \cdots\right)} \\
& =\int_{C}^{W} \Psi_{\mu_{1}, \cdots, \mu_{\nu}}(x) \Psi_{m_{1}, \cdots, m_{n}}\left(x-\lambda \beta_{k}\right) \exp \left\{2 \tau \lambda \int_{0}^{1} \alpha_{k}(t) \tilde{d} x(t)\right\} d_{W} x \\
& =\exp \left\{\tau^{2} \lambda^{2} \int_{0}^{1}\left[\alpha_{k}(t)\right]^{2} d t\right\} \int_{C}^{W} \Psi_{\mu_{1}, \cdots, \mu_{\nu}}\left(x+\lambda \beta_{k}\right) \Psi_{m_{1}, \cdots, m_{n}}(x) d_{W} x \\
& =e^{\tau^{2} \lambda^{2}} \int_{C}^{W}\left\{\prod_{j=1, j \neq k}^{\nu} H_{\mu_{j}}\left[\int_{0}^{1} \alpha_{j}(t) \tilde{d} x(t)\right]\right\} \\
& \cdot H_{\mu_{k}}\left[\int_{0}^{1} \alpha_{k}(t) \tilde{d} x(t)+\lambda \tau\right] \\
& \left\{\prod_{i=1}^{n} H_{m_{i}}\left[\int_{0}^{1} \alpha_{i}(t) \tilde{d} x(t)\right]\right\} d_{W} x \\
& =e^{\tau^{2} \lambda^{2} \pi^{-\nu / 2}} \int_{-\infty}^{\infty} \stackrel{(\nu)}{\cdots} \int_{-\infty}^{\infty}\left\{\prod_{j=1, j \neq k}^{\nu} H_{\mu_{j}}\left(\xi_{j}\right)\right\} H_{\mu_{k}}\left(\xi_{k}+\lambda \tau\right) \\
& \cdot\left\{\prod_{i=1}^{n} H_{m_{i}}\left(\xi_{i}\right)\right\} e^{-\xi_{1}^{2}-\cdots-\xi_{\nu}^{2} d \xi_{1} \cdots d \xi_{\nu}} \\
& =e^{\tau^{2} \lambda^{2}}\left(\prod_{i=1, i \neq k}^{n} \delta_{m_{i}, \mu_{i}}\right)\left(\prod_{j=n+1}^{\infty} \delta_{0, \mu_{j}}\right) \pi^{-1 / 2} \int_{-\infty}^{\infty} H_{\mu_{k}}(\xi+\tau \lambda) e^{-\xi^{2}} d \xi .
\end{aligned}
$$

We now calculate the integral in the last member of (8.4) by a translation and $\mu$ integrations by parts:

$$
\begin{aligned}
\pi^{-1 / 2} \int_{-\infty}^{\infty} H_{\mu}(\xi+\rho) e^{-\xi^{2}} d \xi & =\pi^{-1 / 2} \int_{-\infty}^{\infty} H_{\mu}(\xi) e^{-(\xi-\rho)^{2}} d \xi \\
= & (-1)^{\mu} 2^{-\mu / 2}(\mu !)^{-1 / 2} e^{-\rho^{2}} \pi^{-1 / 2} \int_{-\infty}^{\infty}\left(\frac{d^{\mu}}{d \xi^{\mu}} e^{-\xi^{2}}\right) e^{2 \rho \xi} d \xi \\
& =2^{-\mu / 2}(\mu !)^{-1 / 2}(2 \rho)^{\mu} e^{-\rho^{2}} \pi^{-1 / 2} \int_{-\infty}^{\infty} e^{-\xi^{2}+2 \rho \xi} d \xi \\
& =(\mu !)^{-1 / 2}\left(2^{1 / 2} \rho\right)^{\mu}
\end{aligned}
$$

From (8.4) and (8.5) we obtain (keeping $m_{k}=0$ ),

$$
D_{\mu_{1}}, \ldots, \mu_{\nu}=\left(\prod_{i=1, i \neq k}^{n} \delta_{m_{i}, \mu_{i}}\right)\left(\prod_{j=n+1}^{\nu} \delta_{0, \mu_{j}}\right)\left(\mu_{k} !\right)^{-1 / 2}\left(2^{1 / 2} \tau \lambda\right)^{\mu_{k}} e^{\tau^{2} \lambda^{2}} .
$$

Upon combining (8.3) and (8.6) we have (dropping the $k$ on the one remaining index $\mu_{k}$ ), 


$$
\begin{aligned}
\exp \left\{2 \lambda \tau \int_{0}^{1} \alpha_{k}(t) \tilde{d x}(t)\right\} \Psi_{m_{1}, \cdots, m_{k-1}, 0, m_{k+1}, \cdots, m_{n}}\left(x-\lambda \beta_{k}\right) \\
\sim \sum_{\mu=0}^{\infty}(\mu !)^{-1 / 2}\left(2^{1 / 2} \tau \lambda\right)^{\mu} e^{\tau^{2} \lambda^{2}} \Psi_{m_{1}, \cdots, m_{k-1}, \mu, m_{k+1}, \cdots, m_{n}}(x) .
\end{aligned}
$$

Substitution of (8.7) into (8.2) yields

$$
\begin{aligned}
& B_{m_{1}, \cdots, m_{k-1}, 0, m_{k+1}, \cdots, m_{n}} \\
& \quad=\sum_{\mu=0}^{\infty}(\mu !)^{-1 / 2}\left(2^{1 / 2} \tau \lambda\right)^{\mu} \int_{C}^{W} F(x) \Psi_{m_{1}, \cdots, m_{k-1}, \mu, m_{k+1}, \cdots, m_{n}}(x) d_{W} x \\
& =\sum_{\mu=0}^{\infty}(\mu !)^{-1 / 2}\left(2^{1 / 2} \tau \lambda\right)^{\mu} A_{m_{1}, \cdots, m_{k-1}, \mu, m_{k+1}, \cdots, m_{n} .}
\end{aligned}
$$

But the $B$ 's are the F.H. coefficients of (3.2), and therefore equal to the F.H. coefficients of the right member, so that we have from (8.8),

$$
\begin{aligned}
A_{m_{1}}, \cdots, m_{k-1}, 0, m_{k+1}, \cdots, m_{n} & +\lambda K\left(\prod_{i=1}^{n} \delta_{0, m_{i}}\right) F\left(\beta_{k}\right) \\
& =\sum_{\mu=0}^{\infty}(\mu !)^{-1 / 2}\left(2^{1 / 2} \tau \lambda\right)^{\mu} A_{m_{1}, \cdots, m_{k-1}, \mu, m_{k+1}, \cdots, m_{n}} .
\end{aligned}
$$

As by hypothesis the set of $\lambda$ 's for which (8.9) holds has a limit point within the circle of convergence of the right member, it follows that the coefficients of like powers of $\lambda$ on the two sides of (8.9) must be equal. Comparing coefficients in (8.9), we see that $A_{m_{1}, \cdots, m_{n}}=0$ if the $k$ th subscript (that is, any subscript) is greater than 1 . Upon setting $\mu=1$ and recalling that $m_{k}=0$, we see further that

$$
2^{1 / 2} \tau A_{m_{1}, \cdots, m_{k-1}, 1, m_{k+1}, \cdots, m_{n}}=K\left(\prod_{i=1}^{n} \delta_{0, m_{i}}\right) F\left(\beta_{k}\right),
$$

so that $A_{m_{1}, \cdots, m_{k-1}, 1, m_{k+1}, \cdots, m_{n}}=0$ unless $m_{1}=\cdots=m_{k-1}=m_{k+1}=\cdots=m_{n}$ $=0$. In this special case,

$$
A_{0, \cdots, 0,1}=\left(2^{1 / 2} \tau\right)^{-1} K F\left(\beta_{k}\right),
$$

where there are $k-1$ zeros in the subscript of the $A$, and from which (3.3) follows if we take account of the fact that $H_{1}(u)=2^{1 / 2} u$; and (3.4) follows from (8.10) by the same reasoning.

\section{BIBLIOGRAPHY}

1. R. H. Cameron and W. T. Martin, An expression for the solution of a class of non-linear integral equations, Amer. J. Math. vol. 66 (1944) pp. 281-298.

2. - Transformations of Wiener integrals under translations, Ann. of Math. vol. 45 (1944) pp. 386-396. 
3. - The Wiener measure of Hilbert neighborhoods in the space of real continuous functions, Journal of Mathematics and Physics vol. 33 (1944) pp. 195-209.

4. - The orthogonal development of non-linear functionals in series of Fourier-Hermite functionals, Ann. of Math. vol. 48 (1947) pp. 385-392.

5. Ross E. Graves, Integral representations of linear and weak linear functionals defined over the Wiener space $C$, doctoral dissertation (unpublished), University of Minnesota, 1948.

6. A. Kolmogoroff, Über die Summen durch den Zufall bestimmter unabhängiger Grössen, Math. Ann. vol. 99 (1928) pp. 309-319.

7. R. E. A. C. Paley, N. Wiener, and A. Zygmund, Notes on random functions, Math. Zeit. vol. 37 (1933) pp. 647-668.

8. N. Wiener, Generalized harmonic analysis, Acta Math. vol. 55 (1930) pp. 117-258, especially pp. 214-224.

9. G. Maruyama, Notes on Wiener integrals, Kodai Math. Seminar Reports, No. 3, June 1950, pp. 41-44.

The University of Minnesota, MiNNEAPOLIS, MiNN. 